\title{
DESCRIÇÃO DO PERFIL EPIDEMIOLÓGICO DA COVID-19 NA REGIÃO SUL DO BRASIL
}

\section{DESCRIPTION OF THE EPIDEMIOLOGICAL PROFILE OF COVID-19 IN SOUTHERN BRAZIL}

\author{
Luis Felipe Dias Lopes \\ Doutor em Engenharia de Produção e Sistemas \\ Universidade Federal de Santa Maria \\ Iflopes67@yahoo.com.br \\ Rivaldo Mauro de Faria \\ Doutor em Geografia \\ Universidade Federal de Santa Maria \\ rivaldo.faria@ufsm.br \\ Mauren Pimentel Lima \\ Mestre em Administração \\ Universidade Federal de Santa Maria \\ maurenlima@hotmail.com \\ Raquel Soares Kirchhof \\ Mestre em Enfermagem \\ Universidade Regional Integrada \\ rakel kirch@hotmail.com \\ Damiana Machado de Almeida \\ Doutora em Administração \\ Universidade Federal de Santa Maria (UFSM) \\ adm.damiana@gmail.com \\ Gilnei Luiz de Moura \\ Doutor em Administração \\ Universidade Federal de Santa Maria (UFSM) \\ mr.gmoura.ufsm@gmail.com
}

\begin{abstract}
RESUMO
Objetivos: Descrever o perfil epidemiológico da COVID-19 na Região Sul do Brasil, a partir do seu primeiro caso até o dia 06 de maio de 2020, e verificar como os indicadores brasileiros estão se comportando em relação às metas da OMS para sua prevenção. Método: Estudo transversal, descritivo, retrospectivo e quantitativo. Utilizaram-se porcentagem de cura, coeficiente de detecção de novos casos diagnosticados, de prevalência, de detecção e a taxa de expansão territorial. Fez-se uso de estatística descritiva e análise comparativa entre os estados da Região Sul do Brasil. Resultados: A COVID-19 teve seu início nas capitais dos estados, expandindo-se pelas suas principais rodovias. A projeção de casos da doença foi maior em Santa Catarina (1.209 casos), Paraná (1.140 casos), e menor no Rio Grande do Sul (1.096 casos). Conclusão: Há permanente expansão de casos da COVID-19 na Região Sul, sendo fundamental a manutenção das metas de prevenção da OMS pela população.
\end{abstract}

Palavras-chave: Expansão epidemiológica. Epidemia pelo novo coronavírus. Prevenção e Controle.

\section{ABSTRACT}

Objectives: To report the epidemiological profile of covid-19 in the South Brazilian region from its first case until May 6, 2020 and to verify how Brazilian indicators are behaving in relation to WHO goals for its prevention. Method: Cross-sectional, descriptive, retrospective and quantitative study. We used a percentage of cure, detection coefficient of new

Recebido em: 15/05/2020

Aceito para publicação em: 18/08/2020. 
diagnosed cases, prevalence, detection and rate of territorial expansion. He used descriptive statistics and comparative analysis among the states of southern Brazil. Results: Covid-19 began in state capitals, expanding along its main highways. The projection of cases of the disease was higher in Santa Catarina (1,209 cases), Paraná (1,140 cases), and lower in Rio Grande do Sul (1,096 cases). Conclusion: There is a permanent expansion of cases of covid-19 in the South region, and it is essential to maintain the WHO prevention goals by the population.

Keywords: Epidemiological expansion. Epidemic by the new coronavirus. Prevention and Control.

\section{INTRODUÇÃO}

Ao findar dezembro de 2019, foram registrados os primeiros casos de surto de pneumonia grave, inicialmente, não diagnosticável, na cidade de Wuhan, província de Hubei, na China. Tratava-se de uma nova síndrome respiratória aguda grave ocasionada por um novo vírus (coronavírus 2 ou SARSCOV-2) que, quando em humanos, recebe o nome de COVID-19 (BRASIL, 2020a; XIAO H, ZHANG Y, KONG D, LI S, YANG N., 2020; VERITY et al., 2020).

A sua rápida capacidade de propagação é uma característica preocupante que ocorre pelo contato pessoa a pessoa e com alta capacidade em contaminar objetos inanimados e superfícies, nos quais pode permanecer por 24 horas ou mais (BRASIL, 2020 b).

Tal poder de propagação pode ser observado desde seu surgimento na China (83.974 casos), bem como, pelos surtos posteriores em vários países do mundo, como os EUA (1.252.911 casos), Espanha (221.447 casos), Itália (215.858 casos) e Reino Unido (207.973 casos), sendo estes significativamente maiores em comparação ao seu local de origem (JOHNS HOPKINS UNIVERSITY \& MEDICINE, 2020).

A contaminação pela COVID-19 segue em expansão geográfica contínua, ocasionando preocupação mundial, o que fez a Organização Mundial da Saúde (OMS), em 11 março de 2020, declarar esta infecção como uma pandemia mundial, após serem notificados mais de 110 mil casos de infectados pela doença e 4 mil óbitos em todos os continentes do mundo (JOHNS HOPKINS UNIVERSITY \& MEDICINE, 2020; GARCIA; DUARTE, 2020).

A OMS define pandemia como sendo uma disseminação global de uma nova doença, e indica que os países devem estar preparados para a possibilidade de uma transmissão generalizada nas comunidades, o que pode exigir modificações nas políticas de viagens, elaboração de planos de quarentenas, cancelamento de eventos públicos e o rápido desenvolvimento de medidas terapêuticas como as vacinas (SOCIEDADE BRASILEIRA DE MEDICINA DE FAMÍLIA E COMUNIDADE, 2020).

Outro agravante dessa doença, consiste na sua forma grave, ou seja, o surgimento de pneumonia severa observada mais frequentemente entre indivíduos idosos e/ou portadores de comorbidades (hipertensos, cardiopatas, diabéticos e imunodeprimidos), portanto, ocorre também na população mais jovem (BRASIL, 2020 c, d).

O período médio de incubação da COVID-19 pode variar entre 5,2 a 12,5 dias, sua transmissão leva em média 7 dias após o início do surgimento dos sintomas (febre, tosse, dor de garganta e muscular, entre outros). Alguns estudos sugerem que pacientes assintomáticos também são responsáveis pela sua transmissão, embora ainda existam controversas a respeito (BRASIL, 2020 d, e).

Vários países do mundo já vivenciam desafios e incertezas em relação à sua vigilância e controle e um dos principais são os picos epidêmicos de cada região onde o vírus se manifesta, devido à ampla diversidade ambiental, social e econômica das populações. Por isso, intervenções não farmacológicas (INFs) estão sendo recomendadas como medidas preventivas da COVID-19 e buscam, como metas pautadas na OMS, inibir a sua transmissão, desacelerar seu contágio, diminuir/adiar o crescimento da curva epidêmica e demandas imediatas de saúde (GARCIA, DUARTE, 2020; VILLELA, 2020; KEELING, ROHANI; 2007).

Desse modo, após o surgimento de casos de transmissão comunitária de COVID-19, nas cidades do Rio de Janeiro e São Paulo, no Brasil, em março de 2020, a infecção foi declarada como uma

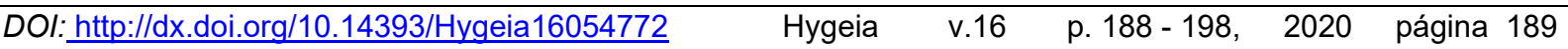


emergência de saúde pública de caráter nacional. Segundo fontes oficiais, em apenas duas semanas houve a confirmação de 47 óbitos pela infecção e de mais 2.200 novos casos do novo coronavírus (BRASIL f, 2002; KRITSKI, WERNECK, ZIMMERMANN, SANCHEZ, GALLIEZ, MEDRONHO, 2020).

No dia 06 de maio de 2020, o número de casos confirmados da COVID-19, no Brasil, totalizava 125.218 e, destes, 8.536 dos infectados foram a óbito; mostrando que a letalidade pelo vírus era de $6,82 \%$ (BRASIL, 2020 c). A maior concentração de casos pelo vírus ocorreu na Região Sudeste $(44,9 \%)$, em relação às demais regiões do país, em que correspondia a: Nordeste $-31,2 \%$; Norte 15,8\%; Sul - 5,1\%; e Centro-Oeste - 3,0\% (BRASIL, 2020 c).

A evolução da COVID-19 ainda é incipiente, o que requer a intensa mobilização por parte da população, pois suas consequências podem ser drásticas em diferentes cidades do país (KRITSKI et al., 2020; SILVA, 2020).

Uma situação não descartada, a exemplo do que já ocorreu em outros países (e.g., Itália), é a probabilidade de aumento da demanda por serviços de saúde, em especial, por leitos em unidade de terapia intensiva (UTI) para o fornecimento de suporte ventilatório nos casos de síndrome respiratória aguda grave, sobretudo nas regiões mais afetadas pela pandemia (RACHE et al., 2020).

Assim, faz-se necessária uma avaliação criteriosa das áreas com maior número de casos da COVID19, a fim de que se possa alocar e utilizar de maneira mais eficiente os recursos e serviços de saúde disponíveis, com capacidade de fornecer uma proposta condizente à situação atual, tanto em âmbito nacional, quanto local (RACHE et al., 2020).

O presente estudo tem como objetivo descrever o perfil epidemiológico da COVID-19 na Região Sul do Brasil, a partir do seu primeiro caso até o dia 06 de maio de 2020, primeiros 65 dias da pandemia, e verificar como os indicadores brasileiros estão se comportando em relação às metas da OMS para sua prevenção.

\section{METODOLOGIA}

Esta pesquisa trata de um estudo transversal, descritivo, retrospectivo e quantitativo. Considerou-se desde o primeiro caso da COVID-19 na Região Sul do Brasil (Paraná (PR), Santa Catarina (SC) e Rio Grande do Sul (RS)), no período de 01 de março de 2020 a 06 de maio de 2020, com uma previsão de casos e óbitos para os 30 dias subsequentes, ou seja, até o dia 05 de junho de 2020.

Os dados foram coletados a partir de informações disponíveis nos seguintes websites: Johns Hopkins University \& Medicine (coronavirus.jhu.edu), Coronavírus Brasil do Ministério da Saúde do Brasil (https://covid.saude.gov.br) e Coronavírus RS do Estado do Rio Grande do Sul (https://ti.saude.rs.gov.br/covid19).

Delimitou-se o período de coleta de dados de 01 de março de 2020, por ter sido a data de registro do primeiro caso na região pesquisada, até a data de fechamento do presente estudo, 06 de maio de 2020, contabilizando os 65 primeiros dias de pandemia na Região Sul do Brasil. No portal nacional (https://covid.saude.gov.br) os dados estão disponíveis em planilha CSV.

O estudo faz parte do projeto "Enfrentamento da epidemia da COVID-19 no estado do Rio Grande do Sul", registrado no CEP/CONEP, CAAE $n^{\circ}$. 130710520.6.0000.5346, CONEP sob o Parecer $n^{\circ}$. 4.003.866.

Para a classificação da endemicidade da COVID-19 foram utilizados porcentagem de cura, coeficientes de prevalência, de detecção, de detecção de novos casos diagnosticados e taxa de expansão territorial.

A análise dos casos confirmados e dos casos de óbito foram realizadas por meio de indicadores e comparativos entre os estados da Região Sul do Brasil, a partir do primeiro caso detectado por estado. Se fez uso de estatística descritiva e comparativa entre os diferentes estados da Região Sul do Brasil, utilizando o programa Office Excel 365. Os gráficos de previsão foram elaborados utilizando técnicas de modelagem epidemiológica (SIR) desenvolvidas por McKendrinck e Kernack (1927) empregando os recursos do (website Insigt Maker (https://insightmaker.com/). Supondo que a probabilidade de infecção para cada contato seja $p$., assim, se tem que o termo: 


$$
p * \beta_{1}
$$

representa a capacidade de um indivíduo infectado transmitir o vírus para outros indivíduos, ou seja, pode apenas infectar indivíduos suscetíveis,. Logo, o termo da capacidade de infecção $(\mathrm{Cl})$ é dado por:

$$
C I=\frac{p * \beta_{1}^{*} S}{T}
$$

onde $T=S+I+R$ representa a população total $(T)$, ou seja, vem a ser a soma dos indivíduos suscetíveis $(S)$, infectados $(I)$ e recuperados $(R)$. Portanto, o número de casos novos é dado por:

$$
N C N=\frac{p^{*} \beta_{1} * S}{T} * I
$$

sendo conhecido como incidência da doença, representando a transferência dos indivíduos suscetíveis para a o grupo de infectados. Quando se tem a taxa de contato proporcional ao número total de indivíduos, ou seja,

$$
\beta_{1}=c_{1} * T \operatorname{com} c_{1} \in \mathfrak{R},
$$

se obtém a taxa de incidência $\delta^{*} . S^{*}$. I, onde $\delta=p^{*} . c_{1}$ é chamado de coeficiente de transição e $\delta$ * $I$ é a força de incidência.

Desse modo, este modelo se torna mais robusto, pois, se a população aumentar, o número de contatos entre os indivíduos será maior, logo, a taxa de contato aumentará. Contrapondo-se, se a população total diminuir, o contato entre os indivíduos também será reduzido, tornando-se uma taxa de contato reduzida. Agora, se considerarmos a taxa de contato sempre constante, isto é $\beta_{1}=c_{2}$ com $c_{2} \in R$, então a incidência é dada por:

$$
I P=\frac{\beta * S^{*} I}{T}, \text { onde } \beta=p^{*} c_{2}
$$

onde IP é chamada incidência padrão, ou também força de infecção (ISEA, LONNGREN, 2013).

O mapa da dispersão da COVID-19 (Figura 1) foi organizado utilizando-se os recursos do software Arcgis, versão 10.8. Se fez uso da interpolação pela Ponderação do Inverso da Distância (IDW), que determina os valores das células usando uma combinação ponderada linearmente de um conjunto de pontos de amostra. O peso é uma função da distância inversa. Após aplicação, classificou-se em 24 classes distribuídas usando o método quantil (DRUCK, CARVALHO, CÂMARA, MONTEIRO, 2004). Como base cartográfica do IBGE, se fez uso da escala 1:200.000, em sistema de projeção Sirgas, 2000.

\section{ANÁLISE DOS RESULTADOS}

Constatou-se, com base nos relatórios diários emitidos pelo Ministério da Saúde, que, nos estados da Região Sul, o estado que apresentou maior número de indivíduos infectados pela COVID-19 foi Santa Catarina (SC) com 2.917 casos confirmados, seguido do Rio Grande do Sul (RS) com 2.050 casos e Paraná (PR) com 1.630 casos.

Referente aos casos de óbito confirmados por essa infecção, o Estado do PR apresentou maior número de óbitos com 101 casos, seguido do RS com 82 e, por fim, SC com 59 casos. Quanto aos casos de letalidade pela COVID-19, a taxa (número de casos de óbito pelo número de pessoas contaminadas) na Região Sul foi de 3,74\%; e, por estado, aquele que apresentou a maior taxa de letalidade para a infecção foi o PR $(6,2 \%)$, seguido do RS (4.24\%), já SC teve a menor taxa de letalidade $(2,02 \%)$ para a infecção até o fechamento desta pesquisa.

A taxa de incidência da COVID-19 no Brasil é de 59,77\%, (número de casos confirmados / número de pessoas com possível risco de contaminação). Destes, $22,67 \%$ correspondem à Região Sul, com

DOI: http://dx.doi.org/10.14393/Hygeia16054772 $\quad$ Hygeia $\quad$ v.16 $\quad$ p. 188-198, 2020 página 191


maior taxa de incidência (43,36/100.000 hab.) no Estado de SC, seguido do RS (22,67/100.000 hab.), e o PR apresentou a menor taxa de incidência para a infecção (14,71/100.000 hab.).

Na Figura 1 apresenta-se a dispersão da pandemia da COVID-19 na Região Sul para os primeiros 65 dias de contaminação.

Figura 1 - Dispersão da COVID-19 na Região Sul nos primeiros 65 dias de pandemia

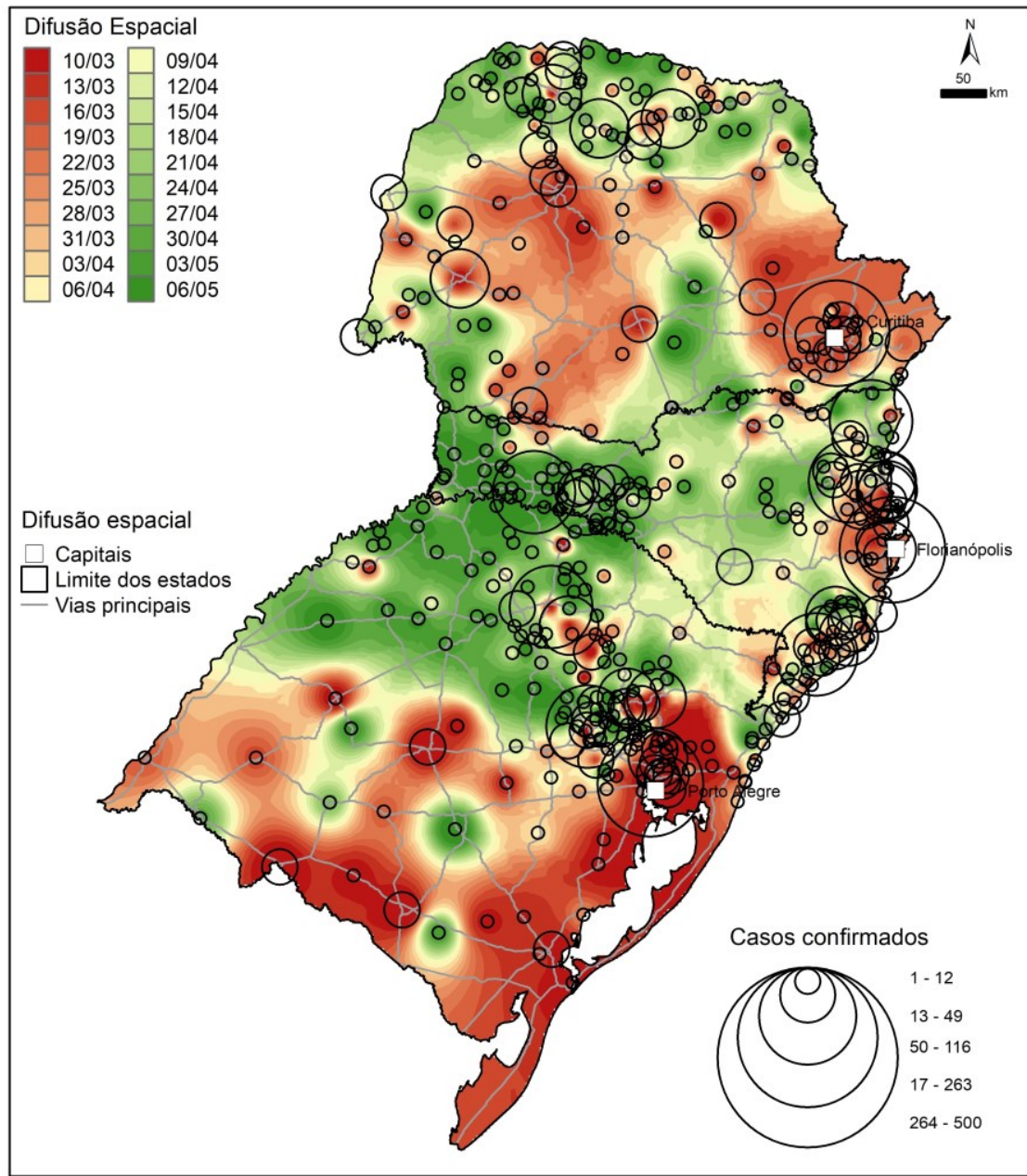

Fonte: Desenvolvida pelos pesquisadores (2020)

Na Figura 1 é ilustrada a expansão da pandemia da COVID-19 com base nas principais rodovias das três regiões que compõem a Região Sul do país.

A primeira região analisada foi o RS, pela BR116 a contaminação da COVID-19 atingiu a cidade de Caxias do Sul (59 casos), polo turístico e industrial, a qual faz parte da serra gaúcha. Na Pela RS453, o maior número de casos pela COVID-19 ocorreu em Bento Gonçalves (94 casos). Por meio da BR101, o vírus propagou-se de Porto Alegre em direção ao litoral, chegando à cidade de Torres (25 casos), cidade turística e litorânea que faz fronteira com SC. Pela RS324, a dispersão da COVID-19 ocorreu de Caxias em direção a Passo Fundo (231 casos), segunda cidade com maior número de casos no Estado do RS, avançando em direção ao norte do RS, e infectou uma parte significativa da cidade de Marau (106 casos). Próxima da cidade de Passo Fundo tem a cidade de Carazinho (16 casos). Através RS287, Passo Fundo faz ligação a Santa Maria (31 casos). Pela BR386, Porto Alegre liga-se a Lajeado (175 casos), atualmente a terceira cidade com maior número de casos no RS. 
Quanto à fronteira oeste do RS, percebeu-se que a infecção por COVID-19 iniciou na cidade de Bagé (29 casos), fronteira com o Uruguai, onde um médico da cidade viajou para a Europa no verão, se contaminou e, ao retornar, contaminou outros quatro médicos (GAÚCHA ZH, 2020). Pela BR293, Bagé faz ligação à cidade de Sant'Ana do Livramento (19 casos), também fronteira com o Uruguai. Pela BR116, a dispersão da COVID-19 ocorreu de Porto Alegre ao porto de Rio Grande (8 casos) e se alastra atingindo a cidade de Pelotas (23 casos).

Pela BR290, ao norte da fronteira oeste do RS, os municípios que apresentam casos confirmados são Alegrete (3 casos) e Uruguaiana (4 casos), ambos ligados com a capital, Uruguaiana, que vem a ser uma cidade fronteira com a Argentina.

Por fim, quanto ao noroeste do estado, ainda tem poucos casos, mas, até o dia 6 de maio, pode-se destacar o Município de Três Passos (30 casos).

Em Santa Catarina, observa-se que a pandemia se concentra no curso da BR101, ou seja, começa por Torres/RS, fronteira com Sombrio (30 casos), segue por Criciúma (175 casos), Braço do Norte (101 casos), Tubarão (68 casos), Imbituba (28 casos), chegando em Florianópolis. Quanto ao leste do estado, atingiu as praias turísticas de Camboriú (76 casos) e Balneário Camboriú (118 casos), Itajaí (120 casos) e Navegantes (82 casos).

Pela BR470 atinge a cidade de Brusque (56 casos) e Blumenau (284 casos), segunda cidade com maior número de casos de SC. Seguindo pela BR101, mais ao nordeste atinge a cidade de Joinvile (245 casos). Pela BR282, apresentam-se casos nas cidades de Concórdia (117 casos), e Chapecó (210 casos) e São Miguel do Oeste (7 casos).

No Paraná, além dos casos da grande Curitiba, atinge a cidade de Paranaguá ao leste (16 casos), fronteira com o Estado de São Paulo. Pela BR 376 Curitiba se liga a Ponta Grossa (19 casos), União da Vitória pela BR476 (7 casos) e Pato Branco pela BR153 (14 casos), cidade essa localizada na fronteira oeste do PR com a Argentina.

Mais ao centro-norte do Estado do PR, nas cidades de Cascavel (86 casos), Campo Mourão (42 casos), Cianorte (24 casos) e Paranavaí (55 casos), sendo esta última cidade, a terceira maior em número de casos. Na BR277 tem Cascavel (86 casos), segunda maior cidade do PR em número de casos, e Foz do Iguaçu (49 casos), cidade turística, fronteira com o Paraguai.

Na Figura 2 são apresentadas os casos da COVID-19 para os estados da Região Sul do Brasil.

Figura 2 - Número de casos acumulados da COVID-19 nos estados da Região Sul do Brasil

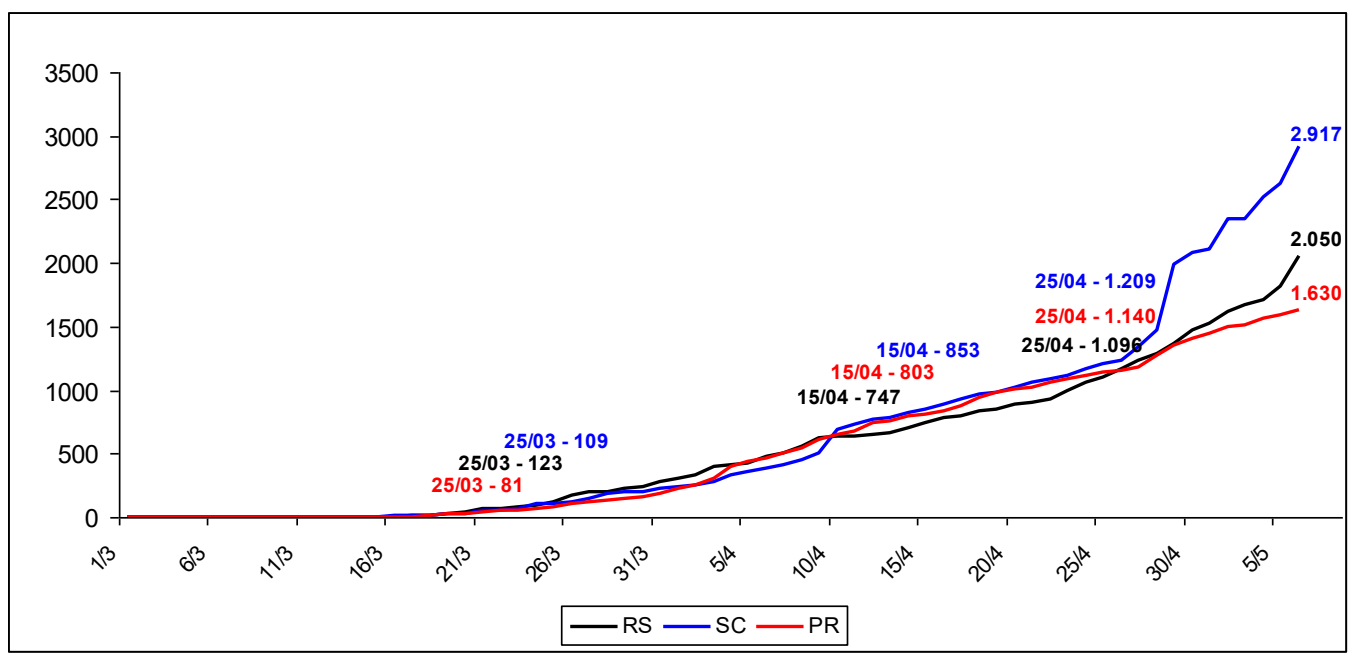

Fonte: Dados da pesquisa (2019)

Na Figura 2, verifica-se que o estado da Região Sul do Brasil que apresentou a o maior número de casos pela COVID-19 até o dia 06 de abril foi SC, passando de 1.209 casos no dia 25 de abril para DOI: http://dx.doi.org/10.14393/Hygeia16054772 $\quad$ Hygeia $\quad$ v.16 $\quad$ p. 188-198, 2020 página 193 
2.917 casos, ultrapassando o dobro de número de casos em 11 dias, seguido do RS, passando de 1.096 casos para 2.050 casos. E o PR foi o estado com menor número de casos pela infecção, passando de 1.096 casos para 1.630, acréscimo de 148,7\%. Na Figura 3 apresentam-se os casos acumulados de óbito na Região Sul pela COVID-19.

Figura 3 - Número de óbitos acumulados pela COVID-19 nos estados da Região do Sul do Brasil

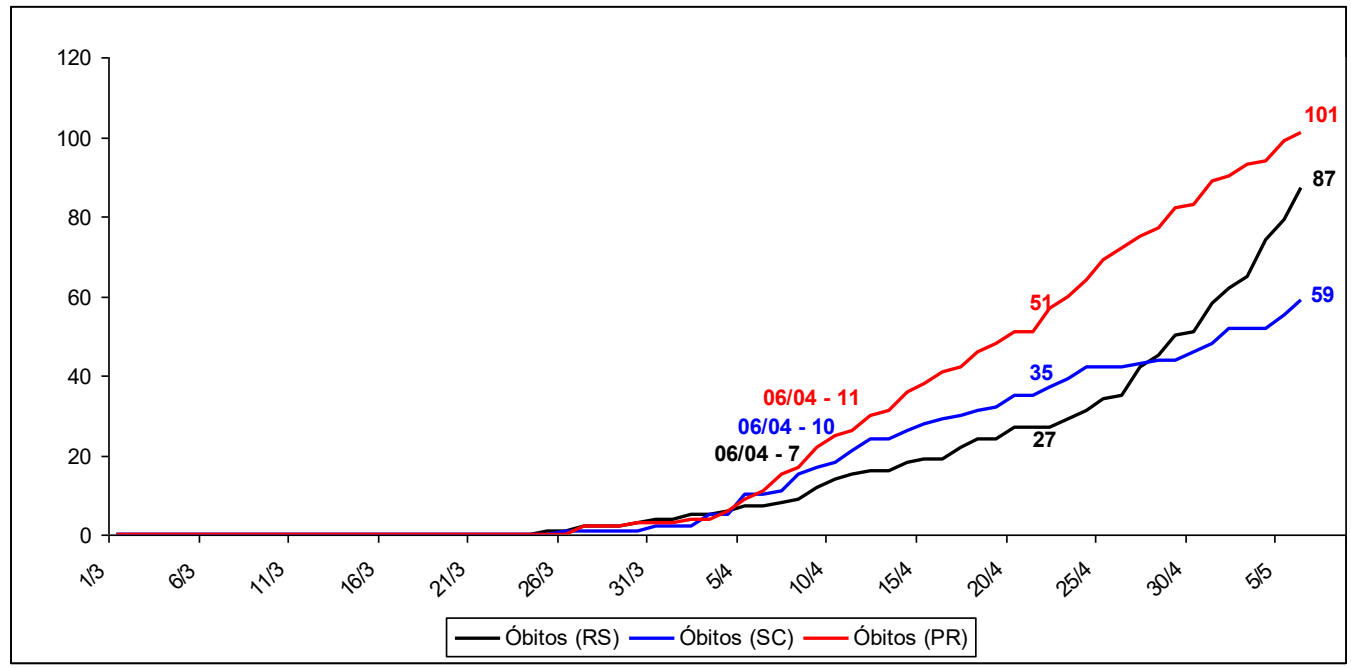

Fonte: Dados da pesquisa (2019)

Na Figura 3, verifica-se que o estado com maior número de óbitos pela COVID-19 durante os 65 dias de contágio foi o PR que, em 11 dias, passou de 51 para 101 óbitos, aumentando para 50 casos, seguido do RS, de 27 para 87 óbitos (60 casos) e o que teve a maior taxa de crescimento. E o estado com menor número de óbitos foi o de SC, que teve um aumento de 19 para 34 casos (15 óbitos).

Nas Figuras 4 e 5 é apresentada a previsão de casos de contaminação e óbitos pela COVID-19 para os próximos 30 dias. As projeções de novos casos apresentadas nas Figuras 4 e 5 usarão um modelo exponencial proposto por (CROKIDAKIS, 2020; FERGUNSON et al., 2020; READ et al., 2020; LI et al., 2020; GUERRA et al., 2020) cujos elementos que irão compor o modelo são: número de casos susceptíveis (metade da população de cada estado), que é considerada a proporção de pessoas susceptíveis a adquirir a doença, podendo em algumas regiões chegar até $75 \%$ (CROKIDAKIS, 2020); a probabilidade de encontrar uma pessoa susceptível (pessoa susceptível / população); fator de transmissão $(0,05)$; taxa de transmissibilidade de 1 para 3 (LIU et al., 2020; Ferguson 2020) e a projeção de casos novos (infectados e óbitos):

$$
N C N=p(P S) * T t^{*} F t * P I,
$$

onde:

$N C N=$ número de casos novos;

$p(P S)=$ probabilidade de encontrar uma pessoa susceptível;

$T t=$ taxa de transmissão;

$F t=$ fator de transmissão;

$P I=$ população infectada; $\mathrm{e}$

$$
N C O=\frac{F P O^{*} N P I}{N D O}
$$

onde:

Hygeia v.16 p. $188-198,2020$ página 194


NCO = novos casos de óbito;

FPO = fração de pacientes que vão a óbito;

$N P I=$ número de pacientes internados;

$N D O=$ número de dias para ir a óbito.

Figura 4 - Previsão do número de casos infectados pela COVID-19 para a Região Sul do Brasil para os próximos 30 dias (de 7 de maio a 5 de junho)

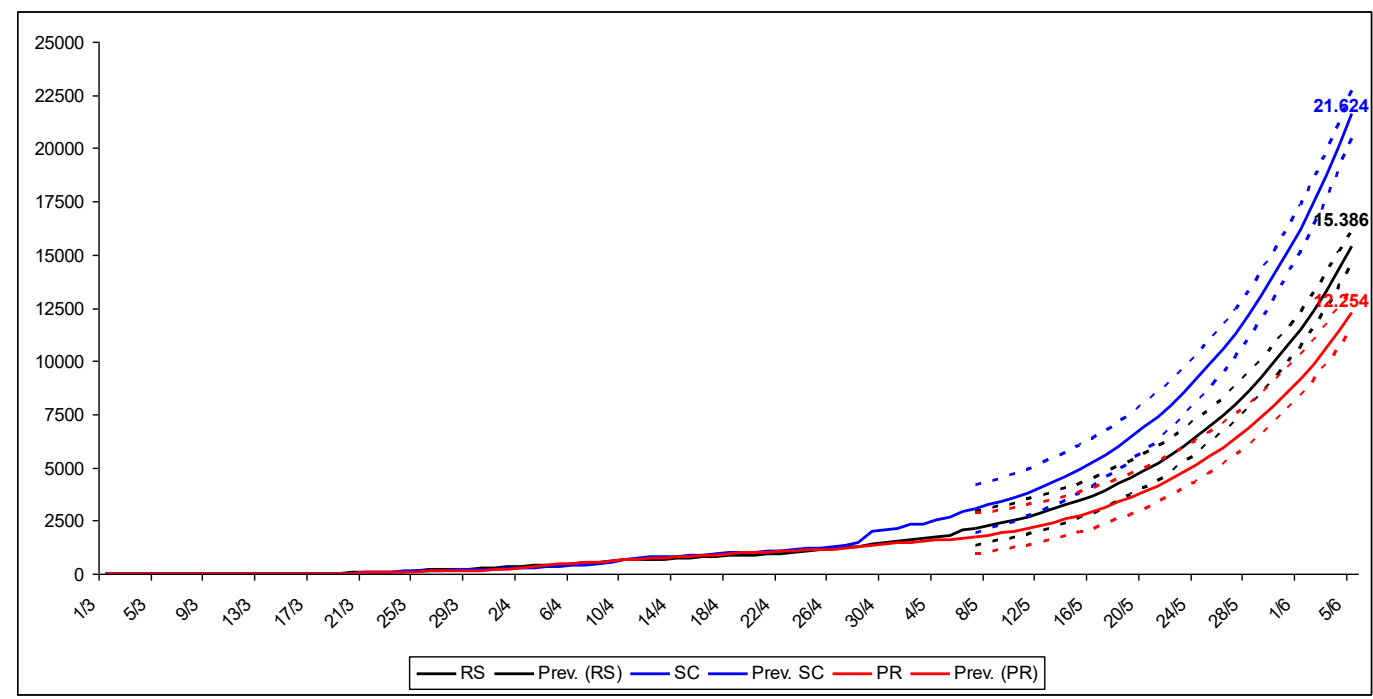

Fonte: Projeções no website Insigt Maker

Por meio da Figura 4 estima-se para SC que o número de pessoas contaminadas por COVID19 para o dia 5 de junho de 2020 seja de 21.624 IC $_{95 \%}(20.509$ a 22.737$)$, para o RS 15.386 IC $_{95 \%}(14.580$ a 16.192) indivíduos e para o PR 12.254 IC $95 \%$ (11.476 a 13.369) para os próximos 30 dias.

Segundo previsões de Kang (2020) e Silva (2020) para os próximos 30 dias, os casos confirmados da COVID-19 indicam que $20 \%$ da população infectada necessitará de internação. Destes, $5 \%$ apresentarão risco de óbito e $1 \%$ irão a óbito, ocorrendo o mesmo com pacientes internados em UTIs, no entanto, a percentagem de óbitos corresponderá a $3 \%$, por serem mais graves.

\section{DISCUSSÃO DOS RESULTADOS}

O crescimento exponencial dos casos da COVID-19 resulta basicamente de sua alta capacidade de infecção, da falta de imunidade prévia das populações e da inexistência de vacinação para o vírus (GARCIA; DUARTE, 2020).

Tal informação vem ao encontro dos dados obtidos neste estudo, os quais descreveram o perfil epidemiológico da Região Sul do Brasil do período inicial da transmissão da doença até o dia 06 de maio de 2020, onde, de um modo geral, identificou-se um aumento progressivo do número de infectados e de óbitos para a região.

No Brasil, até o dia 6 de maio de 2020, havia 125.280 indivíduos infectados pela COVID-19 e 8.536 óbitos (40,7 mortes para cada 1 milhão de habitantes) e uma taxa de letalidade de $6,82 \%$ e $42,7 \%$ dos pacientes curados (RANKBR, 2020).

Em relação aos dados obtidos neste estudo, verificou-se que o número de indivíduos infectados pela COVID-19 no Brasil praticamente dobrou em dez dias, passando de 66.501 casos confirmados para 125.280, e os óbitos, neste mesmo período analisado, passaram de 4.543 para 8.536, seguindo as mesmas projeções. 
A análise dos casos confirmados da COVID-19 por região brasileira, até o dia 06 de maio de 2020, indicou que as regiões com maiores números de infectados foram, respectivamente, a Sudeste (55.843), Nordeste (39.136) e Norte (18.640). As regiões com menor número de infectados a Sul (6.466) e Centro-Oeste (3.621) (RANKBR, 2020).

$\mathrm{Na}$ Região Sul do Brasil, compreendendo a mesma faixa temporal, observa-se que SC permanece sendo o estado com maior número de casos confirmados da COVID-19, com 2.917 infectados, seguido do RS com 2.050 casos. E o PR foi o estado da Região Sul com o menor número de casos, 1.630 indivíduos infectados (CROKIDAKIS, 2020). Tais dados revelam, o crescimento de casos pela COVID-19 nos estados da Região Sul do Brasil entre uma semana e outra, embora, quando comparados aos demais estados brasileiros, tenham uma progressão menor. Tal dado também indica que a incidência de casos é abaixo da média (RANKBR, 2020).

Em relação aos dados obtidos no estudo, verificou-se que a taxa de incidência se manteve abaixo da média em todos os estados, assim como a taxa de letalidade não apresentou crescimento significativo de uma semana para outra, quando esta correspondia a $43,36 \%$ em SC, $18.16 \%$ para o RS e o PR com $14,71 \%$, com um aumento de $6,6 \%$ de uma semana para outra.

Referente à projeção de casos pela COVID-19 no Sul do Brasil para o dia 05 de junho de 2020, o estado que terá o maior número de infectados será SC (21.624 casos), seguido dos estados do RS (15.386 casos) e PR (12.274 casos), este último com o menor número de infectados em relação aos demais estados (RANKBR, 2020). Em comparação à semana do dia 06 de maio, projeta-se, de um modo geral, para todos os estados da Região Sul, um aumento aproximado de sete vezes o número de casos em um mês de evolução da pandemia.

Quanto à projeção de óbitos no Sul do país, até o dia 06 de junho de 2020 estima-se um aumento médio de $230 \%$ no número de casos de óbito pela COVID-19.

\section{CONCLUSÕES}

A presente pesquisa descreveu o perfil epidemiológico da COVID-19 para o Sul do Brasil, verificando os principais indicadores da infecção, os quais sofrem alteração diária. Destaca-se que, ao realizar tal descrição, percebe-se que todos os estados da Região Sul do Brasil apresentaram um aumento de casos pela COVID-19 no período pesquisado. Cabe salientar, no entanto, que, em comparação ao restante do país, as áreas analisadas no estudo ainda possuem um baixo número de casos de infectados e de possíveis óbitos pela COVID-19. Este pode ser um indicativo positivo quanto às metas de prevenção da OMS pela população, embora o estudo aponte a necessidade de estas serem mantidas, com base na dispersão de casos da infecção nas regiões estudadas, havendo a necessidade de manter as metas de prevenção estabelecidas pela OMS. Mas, também, este fato poderá estar associado à subnotificação de casos da doença.

Outro aspecto observado, se refere à maior concentração de casos da COVID-19 nos grandes centros urbanos (capitais e regiões metropolitanas), podendo estar relacionado a diferenças socioeconômicas e demográficas, o que requer uma certa singularidade das medidas pelos seus gestores locais.

As informações utilizadas para a elaboração da pesquisa foram obtidas nas bases de dados oficiais do Ministério da Saúde, e a disponibilização desses dados depende diretamente da atualização do sistema on-line pelas unidades de saúde municipais, estaduais e federais, sendo este um fator limitante do presente estudo, podendo haver a subestimação dos dados quanto à COVID-19.

Além disso, podem ocorrer erros de notificação e diagnóstico tardio que influenciam na descrição do perfil epidemiológico da doença.

E, por fim, ressalta-se que o objetivo principal deste estudo é que a descrição epidemiológica da COVID-19 com base em seus indicadores e que possa oferecer suporte às políticas públicas que visem à prevenção por parte da população, na contenção da disseminação da COVID-19 ou outras pandemias que, em paralelo, possam se desenvolver. 


\section{REFERÊNCIAS}

BRASIL. Ministério da Saúde. Centro de Operações de Emergências em Saúde Pública-COVID19. Secretaria de Vigilância em saúde. Boletim epidemiológico 05. Brasília (Brasil): Ministério da Saúde; 2020a.

Ministério da Saúde. Secretaria de Vigilância em Saúde. Departamento de Análise em Saúde e Vigilância de Doenças não Transmissíveis. Manejo de corpos no contexto do novo coronavírus COVID-19. [Brasília (Brasil): Ministério da Saúde; 2020b.

Ministério da Saúde. Centro de Operações de Emergências em Saúde Pública-COVID19. Secretaria de Vigilância em saúde. Boletim epidemiológico 09. Brasília (Brasil): Ministério da Saúde; 2020c.

Ministério da Saúde. Protocolo de Manejo Clínico para o Novo Coronavírus (2019-nCoV). Brasília (Brasil): Ministério da Saúde; 2020d.

Ministério da Saúde. Secretaria de Atenção Especializada à Saúde. Departamento de Atenção Hospitalar, Domiciliar e de Urgência. Protocolo de manejo clínico da COVID-19 na Atenção Especializada. Brasília (Brasil): Ministério da Saúde; 2020e.

Ministério da Saúde. Secretaria de Vigilância em Saúde. Guia de Vigilância Epidemiológica - Emergência de Saúde Pública de Importância Nacional pela Doença pelo Coronavírus 2019: Vigilância Integrada de Síndromes Respiratórias Agudas Doença pelo Coronavírus 2019, Influenza e outros vírus respiratórios. Brasília (Brasil): 2020f.

CROKIDAKIS N. Data analysis and modeling of the evolution of COVID-19 in Brazil. [cited abr 16, 2020]. Available from: https://arxiv.org/pdf/2003.12150.pdf

DRUCK S.; CARVALHO M. S.; CÂMARA G.; MONTEIRO A. V. M. Análise espacial de dados geográficos. Brasilia: Empresa Brasileira de Pesquisa Agropecuária, 2004.

FERGUSON, N. M.; LAYDON, D.; NEDJATI-GILANI, G.; IMAI, N.; AINSLIE, K.; BAGUELIN, M.; BHATIA, S.; BOONYASIRI, A.; CUCUNUBÁ, Z.; CUOMO-DANNENBURG, G.; DIGHE, A.; DORIGATTI, I.; FU, H.; GAYTHORPE, K.; GREEN, W.; HAMLET, A.; HINSLEY, W.; OKELL, L. C.; ELSLAND, S.; THOMPSON, H.; VERITY, R.; VOLZ, E.; WANG, H.; WANG, Y.; WALKER, P. G. T.; WALTERS, C.; WINSKILL, P.; WHITTAKER, C.; DONNELLY, C. A.; RILEY, S.; GHANI, A. C. Report 9: Impact of non-pharmaceutical interventions (NPIs) to reduce COVID-19 mortality and healthcare demand. Imperial College COVID-19 Response Team. p. 1-20, mar., 2020.

GARCIA L. P.; DUARTE, E. Intervenções não farmacológicas para o enfrentamento à epidemia da COVID-19 no Brasil. Epidemiol. Serv. Saúde. v. 29, i. 2, p. 1-4. 2020. https://doi.org/10.5123/S1679$\underline{49742020000200009}$

GAÚCHA ZH: CORONAVÍRUS SERVIÇO. Cinco entre 11 pacientes com coronavírus em Bagé são médicos. [cited abr 15, 2020]. Available from: https://gauchazh.clicrbs.com.br/coronavirusservico/noticia/2020/03/cinco-entre-11-pacientes-com-coronavirus-em-bage-sao-medicosck8ere08102y201rz2yhhixi0.html

GUERRA F. M.; BOLOTIN, S.; LIM, G.; HEFFERNAN, J.; DEEKS, S. L.; LI, Y; CROWCROFT, N. S. The basic reproduction number (R0) of measles: a systematic review. The Lancet. Infectious Diseases. v. 17, i. 12, p. e420-e428, jul., 2017. https://doi.org/10.1016/S1473-3099(17)30307-9

ISEA, R.; LONNGREN, K E. On the mathematical interpretation of epidemics by kermack and mckendrick. Gen. Math. Notes. v. 19, n. 2, p. 83-87, dec., 2013. Available free online at http://www.geman.in

JOHNS HOPKINS UNIVERSITY \& MEDICINE. COVID-19 Dashboard by the Center for Systems Science and Engineering (CSSE) at Johns Hopkins University (JHU). [cited 25 abr. 2020]. Available from: https://coronavirus.jhu.edu/map.html

KANG, Y. J. Mortality rate of infection with COVID-19 in Korea from the perspective of underlying disease. Disaster Med Public Health Prep. p. 1-3, dec., 2020. https://doi.org/10.1017/dmp.2020.60 
KERMACH, W. O; MCKENDRICK, A. G. A contribution to the mathematical theory of epidemics. In: The Roya Society. Proceedings of the Royal Society of London A: mathematical, physical and engineering sciences. v. 115, i. 772, p. 700-721. 1927. https://doi.org/10.1098/rspa.1927.0118

KEELING, M. J.; ROHANI, P. Modelando doenças infecciosas em humanos e animais. Princeton: Imprensa da Universidade de Princeton; 2007.

KRITSKI, A.; ALVES, D.; WERNECK, G.; ZIMMERMANN, I.; SANCHEZ, M.; GALLIEZ, R.; MEDRONHO, R. Panorama da COVID-19. Nota Técnica de pesquisadores da Universidade Federal do Rio de Janeiro (UFRJ). [cited març 30, 2020]. Available from: https://ufri.br/sites/default/files/imgnoticia/2020/03/notatecnica25032020.pdf.

LI, Q.; GUAN, X.; WU, P.; WANG, X.; ZHOU, L.; TONG, Y. et al. Early Transmission Dynamics in Wuhan, China, of Novel Coronavirus-Infected Pneumonia.The N Engl J Med. v. 382, i. 13, p. 11991207, 2020.

LIU, Y., GAYLE, A. A.; WILDER-SMITH, A.; ROCKLOV, J. The reproductive number of COVID-19 is higher compared to SARS coronavirus. J Travel Med. v. 13, i. 27, 2020. https://doi.org/10.1093/itm/taaa021

RANKBR. Coronavírus a pandemia no Brasil [internet]. [cited abr 25, 2020].

READ, J. M.; BRIDGEN, J. R. E.; CUMMINGS, D. A. T.; HO, A.; JEWELL, C. P. Novel coronavirus 2019-nCoV: early estimation of epidemiological parameters and epidemic predictions. MedRxiv. v. 27 i. 2, p. 1-8, 2020. https://doi.org/10.1101/2020.01.23.20018549

SOCIEDADE BRASILEIRA DE MEDICINA DE FAMÍLIA E COMUNIDADE. [internet] OMS declara pandemia de COVID-19: o que isso significa? [cited abr 01, 2020]. Available from: https://www.sbmfc.org.br/noticias/oms-declara-pandemia-de-coronavirus-o-que-isso-significa/

SILVA, A. M. S. Sobre a possibilidade de interrupção da epidemia pelo coronavírus (COVID-19) com base nas melhores evidências científicas disponíveis. Rev. Bras. Epidemiol. v. 23, i. 2, p. 1-3, mar., 2020. https://doi.org/10.1590/1980-549720200021

VERITY, R.; OKELL, C. L.; DORIGATTI, I.; WINSKILL, P.; WHITTAKER, C.; IMAI, N. et al. Estimates of the severity of coronavirus disease 2019: a model-based analysis. Rev The Lancet. p. 1-9, 2020. https://doi.org/10.1016/S1473-3099(20)30243-7

VILLELA, D. A. M. The value of mitigating epidemic peaks of COVID-19 for more effective public health responses. Rev. Soc. Bras. Med. Trop. v. 53, e20200135, p. 1-2, 2020. https://doi.org/10.1590/0037-8682-0135-2020

XIAO, H.; ZHANG, Y.; KONG, D.; LI, S.; YANG, N. The Effects of Social Support on Sleep Quality of Medical Staff Treating Patients with Coronavirus Disease 2019 (COVID-19) in January and February 2020 in China. Rev Med Sci Monit. v.5, i. 26, e923549-1-e923549-8, mar., 2020. https://doi.org/10.12659/MSM.923921 